\title{
TU4B-(2)-5
}

\section{MODE COMPETITION IN WIDE-RANGE TUNABLE DUAL-WAVELENGTH SEMICONDUCTOR LASER USING NONIDENTICAL INGAASP QUANTUM WELLS}

\author{
Chi-Chia Huang, Chin-Hui Cheng, Yi-Shin Su, and *Ching-Fuh Lin \\ Institute of Electro-Optical Engineering, National Taiwan University, Taipei, Taiwan, Republic \\ of China \\ *also with Institute of Electronics Engineering., and Department of Electrical Engineering \\ Phone: 886-2-23635251 ext 339, Fax: 886-2-23677467, Email: cflin@cc.ee.ntu.edu.tw
}

\begin{abstract}
Different mode competition has been observed in a wide-range tunable dual-wavelength semiconductor laser. When the wavelength separation is large enough, the competition behavior between the two modes is opposite to usual mode competition.
\end{abstract}

Dual-wavelength tunable laser devices are promising components for a wide range of optical applications. Former experiments show that under dual-wavelength operation, some sort of mode competition will occur. However, in our recent experiments with our super-broadband semiconductor optical amplifier (SOA), we have found that when the mode spacing is larger than $110 \mathrm{~nm}$, the light intensity of the long-wavelength mode can be enhanced by the oscillation of the short-wavelength mode, which we called mode anti-competition. Mode anti-competition, joined with the broadband characteristics of the SOA, make it easy for us to obtain wavelength separation larger than $174 \mathrm{~nm}$ under dual-wavelength oscillation.

The dual-wavelength semiconductor laser uses a broadband semiconductor optical amplifier (SOA) as the gain medium. The SOA used in the experiment has nonidentical InGaAsP quantum-well (QW) structure. This QW structure has very broadband characteristics. The semiconductor laser using it as gain medium can be tuned from $1300 \mathrm{~nm}$ to $1540 \mathrm{~nm}$ under single-wavelength operation In the experiment, the SOA is operated at $146 \mathrm{~mA}$ injection current and at temperature of $22.7^{\circ} \mathrm{C}$.

The measurement setup is described as follows. In the external cavity, we use a double slits to choose the two wavelength for oscillation. Two neutral density (ND) filters is placed in front of the double slits to change the light power of each mode. The experiment steps goes through as follows. First, we choose the double silts of certain mode spacing. The grating at the output diffracts the two oscillation wavelengths, and measure their light power through two detectors and an oscilloscope. Next, we tune the ND filter (the one corresponding to the short-wavelength mode) to a certain value of loss and take a measure. This step is repeated until we have tuned the ND filter to its maximum loss, and the light power of the short-wavelength mode is about to vanish.

First, we choose wavelength separation $18.5 \mathrm{~nm}$. We found that there is only competition takes place. However, when the wavelength separation is expanded to $111 \mathrm{~nm}$, the behavior is different. There is a region where the light power of the long wavelength mode increases with the increasing of that of the short-wavelength mode. When the wavelength separation is further expanded to $168.4 \mathrm{~nm}$, the phenomenon of anti-competition is very clear.

The phenomenon of anti-competition is influenced by three factors. The first one is wavelength separation. That is, the larger the wavelength separation is, the more apparent anti-competition will become. We have also done experiment for other wavelength separations, and all of them have the same conclusion. The second factor is the light power of the short-wavelength mode. The phenomenon of anti-competition can exist only when light power of the short-wavelength mode is below certain level. The third factor is the light power of the long-wavelength mode when the short-wavelength mode is vanished.

The possibility of mode anti-competition is due to the following reasons. First, the gain of the nonidentical QWs is broad, and at large mode spacing the two wavelengths are nearly contributed from two different QWs. Therefore the competition between them is weak because the carrier transportation between QWs is a relatively slow process, compared to the intraband relaxation in the same well. Second, because the photon energy of the short-wavelength QWs is larger than that of the long-wavelength QWs, some of the emitted short-wavelength photons will be absorbed by the long-wavelength QWs. This provides the long-wavelength QWs with some optical pumping, in addition to the electrical pumping with which we provide. At large wavelength separation, weak mode competition is suppressed by optical pumping, resulting in the phenomenon of anti-competition. When the wavelength separation is small or the light power of any of the two wavelengths is too large, optical pumping still exists, but now it is suppressed by mode competition.

Using this setup, the maximum wavelength separation we can obtain is $174.6 \mathrm{~nm}$, with short- and long-wavelength mode locate in $1355.6 \mathrm{~nm}$ and $1530.1 \mathrm{~nm}$, respectively. 